\title{
Endometrial Scratch-Suction and Implantation Failure
}

\author{
Wael S. Nossair, Manal M. El Behery, Mohamed Al S. Farag \\ Department of Obsteterics and Gynaecology, Faculty of Medicine, Zagazig University, Zagazig, Egypt \\ Email: wsnossair@gmail.com
}

Received 1 December 2013; revised 2 January 2014; accepted 10 January 2014

Copyright (C) 2014 by authors and Scientific Research Publishing Inc.

This work is licensed under the Creative Commons Attribution International License (CC BY). http://creativecommons.org/licenses/by/4.0/

(c) (i) Open Access

\begin{abstract}
Objective: The aim of this trial was to determine whether endometrial scratch-suction caused by insertion tube of (IUCD) with suction improved the probability of pregnancy in the subsequent IVF cycle in patients who had previous failed IVF outcome by removing small polyps and any hazardous materials. Design: Observational prospective cohort experimental study. Setting: Zagazig University Hospital, Obstetrics and Gynecology department. From period between September 2011 till September 2012. Population: 30 patients with previous failed IVF despite transfer of goodquality embryos were allocated to endometrial scratch-suction in the same the cycle of embryo transfer. Methods: From day 5 to day 7 of same ICSI cycle, the patients in study underwent endometrial scratch-suction technique. After preparation of insertion tube of (IUCD) and infant feeding tube 8 F, moving the insertion tube of IUCD from below upwards with scratching anterior uterine wall, then posterior, then left lateral uterine wall, then right lateral uterine wall, then fundus, all these steps are associated with suction by attaching proximal end of infant feeding tube $8 \mathrm{~F}$ to suction apparatus. Main Outcome Measures: Implantation and clinical pregnancy rates. Results: The implantation occurred in $24 / 30(80 \%)$. The clinical pregnancy occurred in $20 / 30$ patients $(66.66 \%) .12 / 30$ patients $(40 \%)$ during scratch-suction technique had small fleshy masses obstructing suction tube which were small endometrial polyps. Conclusions: Endometrial scratch-suction improves clinical pregnancy and implantation rates in the same IVF-ET cycle in patients with previous unsuccessful IVF cycles.
\end{abstract}

\section{Keywords}

Endometrial Scratch-Suction; IVF/ICSI; Endometrial Polyps; Chemical Pregnancy; Clinical Pregnancy

\section{Introduction}

The process of implantation still remains a limiting factor in IVF-ET. For implantation to occur, a genetically 
normal blastocyst should hatch, appose, adhere, penetrate, and finally invade a well-synchronized endometrium, under the influence of estrogens and progesterone. A number of locally acting molecules including growth factors, cytokines, matrix metalloproteinases (MMPs), adhesion molecules, extracellular matrix components, and homeobox element containing genes, which mediate the action of the steroids hormones on the endometrium, have been discovered [1] [2].

Implantation failure, which is presently the major barrier in human fertility, is attributed, in many cases, to the failure of the uterus to acquire receptivity. The transition into a receptive uterus includes cellular changes in the endometrium and the modulated expression of different cytokines, growth factors, transcription factors, and prostaglandins. Embryo implantation is associated with an active Th1 inflammatory response while a Th2-humoral inflammation is required for pregnancy maintenance. Yet, the findings suggest that a Th1 inflammatory response is also necessary for the acquisition of uterine receptivity. This notion was originally suggested by reports from many clinical centers worldwide that IVF patients with repeated implantation failure subjected to endometrial biopsy exhibit a substantial improvement in their chances to conceive. These findings, followed by the demonstration of an elevated pro-inflammatory cytokine/chemokine expression, as well as an increased abundance of immune cells, in the endometrium of these patients, raised the idea that the acquisition of uterine receptivity is closely associated with an inflammatory response. No benefit from local injury to the endometrium in women with a high number of RIFs [3].

The treatment of repeated implantation failure in spite of transfer of good-quality embryos continues to be a dilemma. Barash et al. [4] were the first to study the effect of endometrial injury on the pregnancy outcome [5]. They demonstrated a significant doubling of the implantation, clinical pregnancy, and live birth rates in patients who underwent endometrial scraping in the cycle immediately preceding the IVF cycle. They hypothesized that the injury inflicted on the endometrium could lead to a massive secretion of growth factors and cytokines during the process of wound healing, which could help in implantation.

Endometrial injury in nontransfer cycle improves the live birth rate, clinical pregnancy and implantation rates in the subsequent IVF-ET cycle in patients with previous unsuccessful IVF cycles [6] (Figure 1).

\section{Methods}

\subsection{Study Design and Subject Selection}

This is an observational prospective cohort experimental trial, involving patients undergoing ICSI treatment at fertility center. The subjects were recruited from the period between May 2011 and October 2012.

\subsection{Study Participants}

Institutional review board (IRB) approval was taken before the study and patients consent after explanation of

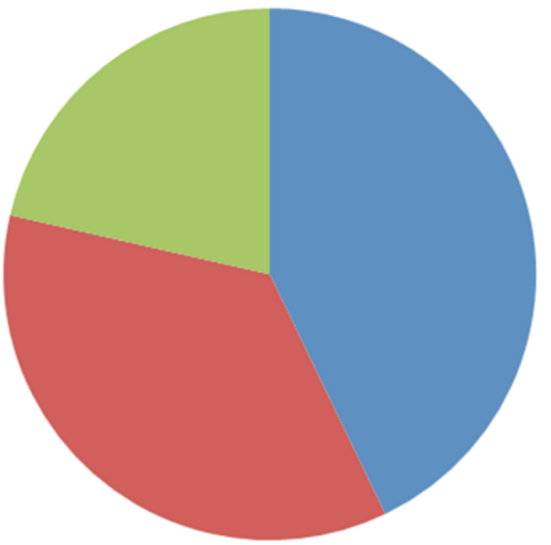

Implantation $\quad$ Clinical pregnancy Endometrial polyps

Figure 1. Implantation, clinical pregnancy and endometrial polyp ratio. 
procedure to them.

We enrolled patients undergoing fresh autologous ICSI-ET, if they fulfilled all of the following inclusion criteria:

1) Patients with at least one previous failed ICSI cycles undergoing fresh autologous ICSI cycles.

2) Good responders in the previous ICSI cycle.

3) Age: Less than or equal to 35 years.

We excluded patients with the following factors found to have a negative impact on implantation, namely:

1) Presence of intramural fibroid distorting the endometrial cavity/submucous myoma/asherman's syndrome.

2) Presence of sonographically detected hydrosalpinx.

3) Patient age more than 35 years.

Good responders were defined as the patients who had developed at least four good-quality embryos (grade 1 and 2 of Veeck's grading) in the previous IVF cycles.

\subsection{Study Design}

Patients found eligible for the study were offered to undergo endometrial scratch-suction in the same cycle of the embryo transfer cycle. After obtaining an informed consent. These patients included in this prospective cross sectional study (Table 1 ).

Institutional review board (IRB) approval was obtained before the study (April 2011, 920).

\subsection{Treatment Protocol}

According to our internal protocol, all patients were evaluated with baseline day $3 \mathrm{FSH}$, and antral follicle count of the cycle prior to the embryo transfer cycle. Records of previous stimulation protocols and embryology details were reviewed.

The patients in study underwent endometrial scratch-suction technique. After preparation of insertion tube of intrauterine contraceptive device (IUCD) and infant feeding tube $8 \mathrm{~F}$ after cutting it’s upper end, and moving the insertion tube of IUCD from below upwards with scrachning anterior uterine wall then posterior then left lateral uterine wall then right lateral uterine wall then fundus, all these steps associated with suction by attaching proximal end of infant feeding tube $8 \mathrm{~F}$ to suction apparatus. All patients were prescribed. Doxycyclin $100 \mathrm{mg}$ twice daily for 3 days starting one day before the procedures. The technique was done from day 5 to day 7 of same ICSI cycle.

Each woman recruited in the study underwent the same $\mathrm{COH}$ protocol that she had undergone in the previous ICSI cycles, which included one of the two regimens, namely, long midluteal phase GnRH agonist suppression, or GnRH antagonist protocols.

The protocols are selected depending on age, antral follicle count, and serum FSH levels. The GnRH agonists midluteal downregulation protocols are preferred for age groups $\leq 35$ years, FSH $<8$ IU/l, and the combined antral follicle count $\geq 10$. In the long protocol, patients were downregulated with $0.5 \mathrm{mg} \mathrm{GnRH}$ agonist for the period of $10-14$ days following which the dose was reduced to $0.2 \mathrm{mg}$ and continued till hCG. After confirming adequate downregulation, FSH, in the dose ranging from 150 to $250 \mathrm{IU}$, was commenced.

In the Antagonist group, flexible, multiple-dose regimens were used. GnRH antagonist was started at a dose of $0.25 \mathrm{mg}$ when at least one follicle reached $14 \mathrm{~mm}$. FSH was continued till ovulation trigger.

Women were scheduled for oocyte retrieval when at least three follicles reached a size of $18 \mathrm{~mm}$. Oocyte retrieval was performed by the transvaginal route under ultrasound guidance, 34-hr after HCG trigger with 5000 IU, with the patient under conscious sedation. The morphology of each aspirated oocyte was noted after denudation with hyaluronidase (Figures 2 and 3). ICSI was performed for all patients [7].

The embryos were classified according to Veeck's grading as follows:

Grade 1: preembryos with blastomeres of equal size and no cytoplasmic fragmentation;

Table 1. Patients included in the study.

\begin{tabular}{cccccccc}
\hline Patient meet criteria & \multicolumn{3}{c}{ Patients refuse } & Patient not meet criteria & \multicolumn{2}{c}{ Total } \\
\hline No. & $\%$ & No & $\%$ & No. & $\%$ & No. & $\%$ \\
30 & $60 \%$ & 5 & 10 & 15 & $35 \%$ & 50 & $100 \%$ \\
\hline
\end{tabular}




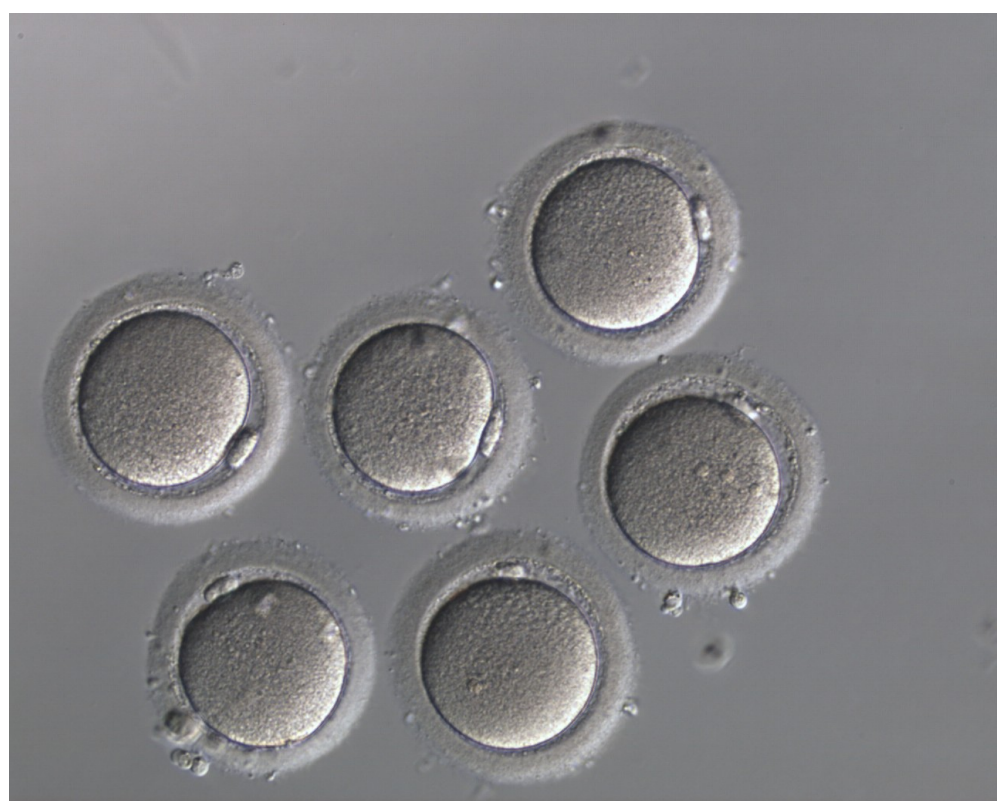

Figure 2. Oocytes M2.

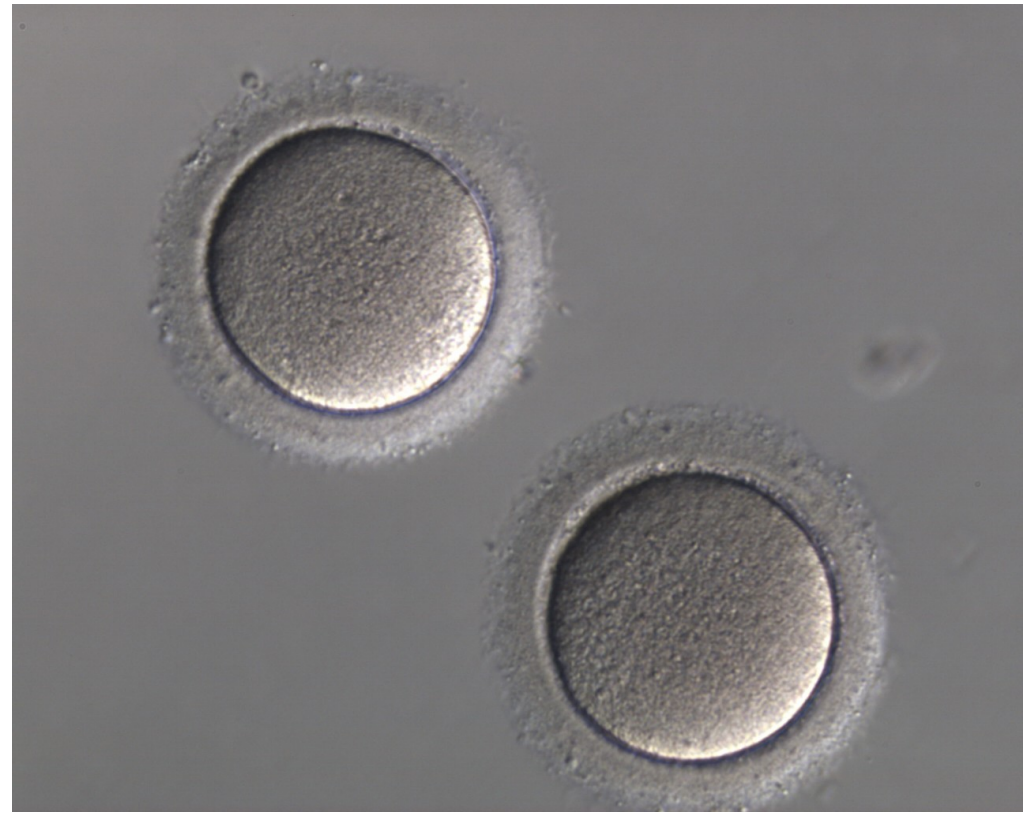

Figure 3. Oocytes M1.

Grade 2: preembryos with blastomeres of equal size with cytoplasmic fragmentation equal to $15 \%$ of the total embryonic volume);

Grade 3: uneven blastomeres with no fragmentation;

Grade 4: uneven blastomeres with gross fragmentation ( $\geq 20 \%$ fragments).

Grade 1 or 2 embryos were considered to be good-quality embryos. Embryos seen at day 3 and ET at day 5.

Embryo transfer was performed with a Wallace ${ }^{\circledR}$ catheter (Smith Medical International Ltd., Hythe, Kent, UK) on day 5 (Figures 4 and 5) a traumatically under ultrasound guidance by a senior consultant. We transfer two to three good-quality embryos. Assisted hatching was not done in any of the patients. Luteal phase was supported with $600 \mathrm{mg} /$ day of micronized progesterone vaginally till 12 weeks of pregnancy. $\beta$-hCG was determined 2 weeks after the embryo transfer (Table 2). 
Table 2. Patient data record; 1: negative, 2: positive.

\begin{tabular}{|c|c|c|c|c|c|c|c|c|c|c|c|}
\hline $\begin{array}{l}\text { Patient } \\
\text { serial }\end{array}$ & Age & Fsh & $\begin{array}{l}\text { Sperm } \\
\text { progressive } \\
\text { motility }\end{array}$ & $\begin{array}{l}\text { No of } \\
\text { failed icsi } \\
\text { cycles }\end{array}$ & $\begin{array}{l}\text { Follicle } \\
\text { number }\end{array}$ & $\begin{array}{c}\text { Oocyte } \\
\text { no. }\end{array}$ & $\begin{array}{c}\text { Blastocst } \\
\text { no. }\end{array}$ & $\begin{array}{l}\text { Number } \\
\text { of embryo } \\
\text { transfer }\end{array}$ & Polyp & Impnataion & $\begin{array}{c}\text { Clinical } \\
\text { pregnancy }\end{array}$ \\
\hline 1 & 32 & 4 & $10 \%$ & 1 & 20 & 16 & 10 & 2 & 1 & 1 & 1 \\
\hline 2 & 30 & 6 & $20 \%$ & 1 & 16 & 14 & 8 & 2 & 1 & 2 & 2 \\
\hline 3 & 26 & 3 & $15 \%$ & 2 & 26 & 24 & 13 & 2 & 2 & 2 & 2 \\
\hline 4 & 29 & 5 & $32 \%$ & 3 & 18 & 18 & 10 & 2 & 1 & 2 & 1 \\
\hline 5 & 28 & 4.5 & $40 \%$ & 1 & 22 & 21 & 12 & 2 & 2 & 1 & 1 \\
\hline 6 & 30 & 5.6 & $5.6 \%$ & 2 & 20 & 20 & 10 & 2 & 1 & 2 & 2 \\
\hline 7 & 28 & 5 & $9 \%$ & & 20 & 19 & 12 & 2 & 1 & 2 & 2 \\
\hline 8 & 26 & 3.9 & $8.4 \%$ & 3 & 26 & 23 & 14 & 2 & 2 & 2 & 2 \\
\hline 9 & 28 & 6.2 & $14 \%$ & 1 & 28 & 25 & 15 & 2 & 1 & 1 & 1 \\
\hline 10 & 26 & 7 & $12 \%$ & 2 & 24 & 22 & 16 & 2 & 1 & 2 & 2 \\
\hline 11 & 27 & 5.7 & $22 \%$ & 3 & 18 & 16 & 12 & 2 & 2 & 2 & 1 \\
\hline 12 & 24 & 6.3 & $25 \%$ & 3 & 16 & 15 & 10 & 2 & 1 & 2 & 2 \\
\hline 13 & 31 & 4.5 & $26 \%$ & 3 & 19 & 19 & 12 & 2 & 2 & 2 & 2 \\
\hline 14 & 30 & 6.1 & $24 \%$ & 2 & 20 & 18 & 11 & 2 & 2 & 1 & 1 \\
\hline 15 & 32 & 5.9 & $21 \%$ & 3 & 24 & 22 & 13 & 2 & 1 & 1 & 1 \\
\hline 16 & 30 & 4.7 & $12 \%$ & 2 & 26 & 25 & 14 & 2 & 1 & 2 & 2 \\
\hline 17 & 31 & 5.6 & $30 \%$ & 1 & 22 & 20 & 14 & 2 & 21 & 2 & 2 \\
\hline 18 & 34 & 5.8 & $10 \%$ & 2 & 24 & 23 & 14 & 2 & 2 & 2 & 2 \\
\hline 19 & 32 & 4.9 & $12.7 \%$ & 3 & 18 & 18 & 12 & 2 & 1 & 2 & 1 \\
\hline 20 & 32 & 4.3 & $20 \%$ & 1 & 16 & 16 & 10 & 2 & 2 & 2 & 2 \\
\hline 21 & 27 & 6.3 & $18.7 \%$ & 2 & 18 & 15 & 10 & 2 & 1 & 2 & 2 \\
\hline 22 & 29 & 4.5 & $19.9 \%$ & 2 & 20 & 19 & 14 & 2 & 2 & 2 & 2 \\
\hline 23 & 32 & 4.8 & $60 \%$ & 2 & 22 & 22 & 16 & 2 & 2 & 1 & 1 \\
\hline 24 & 31 & 4.9 & $20 \%$ & 3 & 20 & 18 & 15 & 2 & 1 & 2 & 2 \\
\hline 25 & 30 & 5 & $32 \%$ & 2 & 22 & 20 & 14 & 2 & 2 & 2 & 2 \\
\hline 26 & 29 & 4.5 & $36.8 \%$ & 1 & 18 & 18 & 14 & 2 & 1 & 2 & 1 \\
\hline 27 & 28 & 6 & $40 \%$ & 2 & 16 & 16 & 12 & 2 & 1 & 1 & 1 \\
\hline 28 & 30 & 4 & $15 \%$ & 3 & 20 & 18 & 12 & 2 & 1 & 2 & 2 \\
\hline 29 & 28 & 5.8 & $16.9 \%$ & 3 & 18 & 17 & 14 & 2 & 1 & 2 & 2 \\
\hline 30 & 27 & 6.2 & $30 \%$ & 2 & 20 & 19 & 15 & 2 & 1 & 2 & 1 \\
\hline
\end{tabular}

\subsection{Outcome Variables}

Outcome measures were implantation and clinical pregnancy rates. The implantation rate was defined as the number of gestational sacs as seen on transvaginal sonography divided by the number of embryos transferred. Clinical pregnancy was defined as ultrasound evidence of fetal heart beat. Clinical pregnancy rate was calculated as the number of patients with clinical pregnancy divided by the number of patients who had embryo transfer (Figure 1).

\subsection{Statistical Analyses}

Statistical analyses were performed using the Statistical Package for the Social Sciences (10.0 SPSS). 


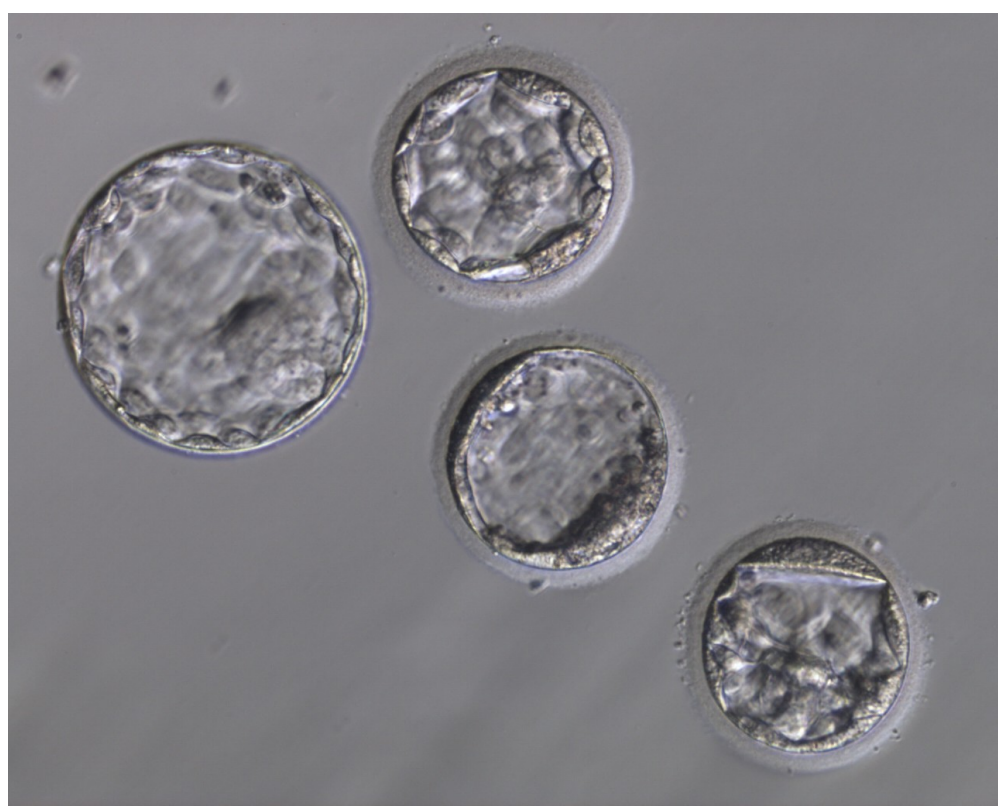

Figure 4. Blastocyst stage (1).

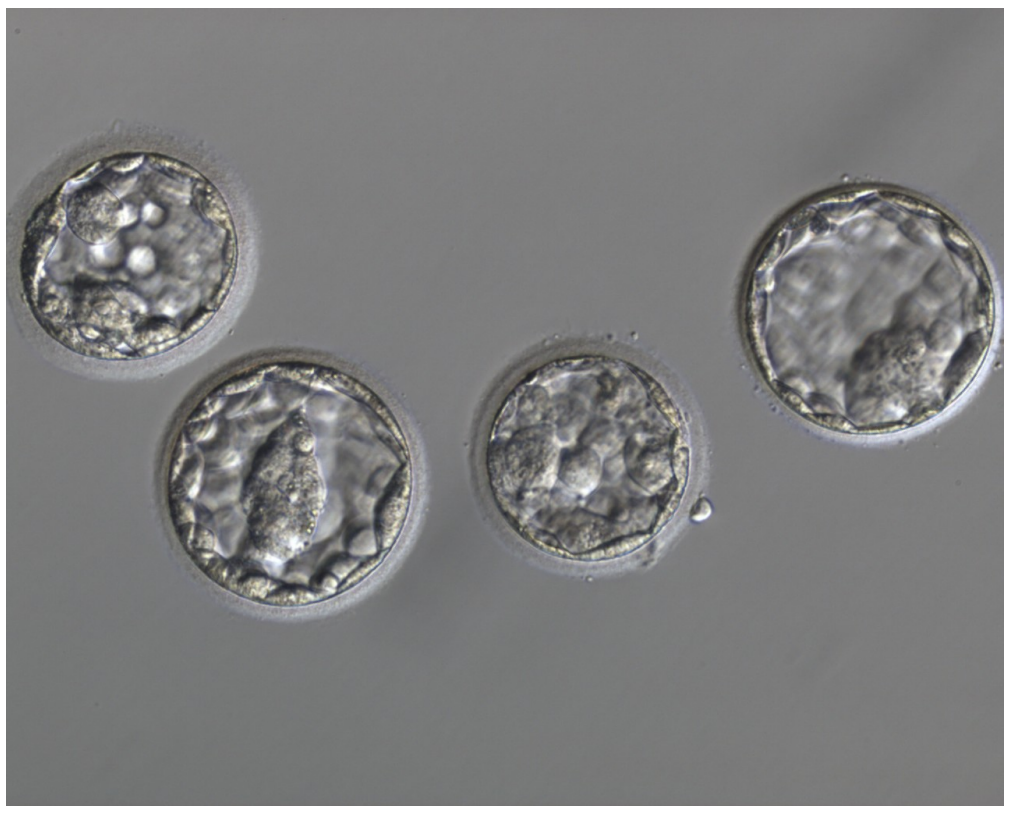

Figure 5. Blastocyst stage (2).

\section{Results}

Out of a total of 50 patients found eligible for recruitment, 20 were excluded either due to refusal to participate $(n=5)$ or failure to meet the inclusion criteria $(n=15)$. Thus 30 patients were included in the study.

\subsection{Baseline Characteristics}

All our patients had primary infertility. The distributions of patients in the two different protocols were not significantly different.

All patients were monitored for the evidence of infection following the scratch suction. None of our patients developed infection. Also, other than spotting per-vagina for one to two days, there was no disturbance in the 
menstrual cycle.

\subsection{Outcome Measures}

The implantation occurred in 24/30 (80\%). The clinical pregnancy occurred in 20/30 patients (66.66\%). 12/30 patients (40\%) during scratch-suction technique small fleshy masses obstructed suction tube and were small endometrial polyps.

There were 5 twins and 3 triplet pregnancy in. There were three spontaneous miscarriages, all after the appearance of fetal pole.

\section{Discussion}

\subsection{Main Findings}

In this observational prospective cohort experimental study trial including 30 patients with previous failed IVF despite transfer of good-quality embryos were allocated to endometrial scratch-suction in the same the cycle of embryo transfer. The implantation occurred in 24/30 (80\%). The clinical pregnancy occurred in 20/30 patients (66.66\%). 12/30 patients (40\%) during scratch-suction technique small fleshy masses obstructed suction tube and were small endometrial polyps. Endometrial scratch-suction improves clinical pregnancy and implantation rates in the same IVF-ET cycle in patients with previous unsuccessful IVF cycles.

\subsection{Strengths and Limitations of the Study}

The major strength of this trial was the observational prospective cohort experimental design, which has, to the best of our knowledge, not previously been used for investigating the association between endometrial-scratchsuction and improving implantation failure. All patients were previously hysteroscopically evaluated with different time regarding the cycle if IVF-ET, but the theoretical suspected risk of new development of endometrial polyps or hazards materials by newly growing endometrium in same cycle of IVF-ET not taken in consideration in many studies in addition to risks of hysteroscopy.

The scientific explanation of the effect of endometrial injury is not yet fully clear. It was observed by Leob in 1907 that scratching the progestational guineapig uterus resulted in decidualization of the endometrium. Later it was observed in rats that injection of oil also resulted in decidualization of progestational uterus [8]-[10].

Clinical and basic science data regarding the association between endometrial injury and improved implantation rate are limited. However, current evidence suggests that endometrial injury before IVF among women with previous repeated IVF failure is associated with increased rates of implantation, clinical pregnancy, and live birth. Endometrial injury may have a beneficial role in implantation and improve the pregnancy rate. However, there are still many unanswered question including patients selection, timing, technique and number of endometrial biopsies needed [11].

Barash et al., 2003 [4]; published a prospective case-control study of 45 "good responder" subjects who failed to conceive during one or more IVF-ET cycles. Endometrial samples were taken on days 8, 12, 21, and 26 of the menstrual cycle prior to their next IVF-ET. They reported a significantly doubled clinical pregnancy rate (66.7\% vs $30.3 \%$ ), implantation rate ( $22.7 \%$ vs $14.2 \%$ ), and live birth rates ( $48.9 \%$ vs $22.5 \%)$.

Subsequently, Raziel et al., 2007 [12]; reported a case control study in a group of 60 ICSI patients with higher order implantation failure (more than four unsuccessful embryo transfers of fresh embryos). The Pipelle biopsy was performed twice on days 21 and 26. They demonstrated an increased implantation (11\% vs $4 \% P=0.02)$, clinical pregnancy ( $30 \%$ vs $12 \% P=0.02$ ), and ongoing pregnancy rates ( $22 \%$ vs $8 \% P=0.07$ ) in the intervention groups.

In a randomised control trial study, 115 women each with at least two implantation failures were randomly assigned to two groups. In the case group, endometrial biopsy was obtained from patients in the luteal phase of previous cycle, and implantation and clinical pregnancy rates were compared with those of patients in the control group. The implantation rate was determined as $10.9 \%$ in the biopsy group compared to $3.38 \%$ in the controls. The clinical pregnancy rate was significantly higher in the case group than in controls $(27.1 \%$ and $8.9 \%$ respectively).The results suggest that pregnancy outcome increases through IVF or ICSI after endometrial biopsy [13]. In this study the endometrial scratch suction done at same cycle. 
Some limitations to this study may apply. In terms of genetic or metabolic abnormalities of the embryo, and endometrial adhesive chemicals evaluation. The study was not powered to evaluate genetic or metabolic abnormalities of the embryo, and endometrial adhesive chemicals.

Approximately half of all human embryo implantations result in failed pregnancy. Multiple factors may contribute to this failure, including genetic or metabolic abnormalities of the embryo. However, many of these spontaneous early abortion cases are attributed to poor uterine receptivity. Furthermore, although many fertility disorders have been overcome by a variety of assisted reproductive techniques, implantation remains the ratelimiting step for the success of the in vitro fertilization (IVF) treatments. It has been demonstrated that endometrial biopsies performed either during the spontaneous, preceding cycle, or during the IVF cycle itself, significantly improve the rate of implantation, clinical pregnancies and live births. These observations suggest that mechanical injury of the endometrium may enhance uterine receptivity by provoking the immune system to generate an inflammatory reaction. In strong support of this idea, we recently found that dendritic cells (DCs), an important cellular component of the innate immune system, play a critical role in successful implantation in a mouse model. The injury-derived inflammation in the biopsy-treated patients generates a focus for uterine DCs accumulation that, in turn, enhances the endometrial expression of essential molecules, which facilitate the interaction between the embryo and the uterine epithelium [14].

Zhou et al., 2008 [15]; performed endometrial scratching, during controlled ovarian hyperstimulation (COH) in 60 patients treated with long protocol, who were good responders to previous hormonal stimulation and had an irregular endometrial echopattern on transvaginal sonography. They demonstrated a significantly increased implantation rate (33.3\% vs $17.7 \% P<0.05)$, clinical pregnancy rate $(48.3 \%$ vs $27.8 \% P<0.05)$, and live birth rates $(41.6 \%$ vs $22.9 \% P<0.05)$. In addition, 10 endometrial biopsy samples obtained on day 10 of the $\mathrm{COH}$ cycle were processed individually for gene chip hybridization. They found a total of 218 genes showing a statistically significant different expression when comparing the pregnant and nonpregnant patients. Of these, 41 were upregulated and 177 were downregulated. The genes for laminin alpha 4 and MMP1 were upregulated, while that of integrin alpha 6 were downregulated. While the exact function of laminin alpha 4 is not known, MMP1 and integrin alpha 6 play an important role in implantation [16] [17]. The same authors also proposed that the injury-induced wound healing could result in the slowing of endometrial development, which could further lead to enhanced embryo-endometrial synchronization.

Kalma et al., 2009 [18]; demonstrated that endometrial injury on days 11 - 13 and 21 - 24 of the nontransfer cycle resulted in upregulation of 183 genes and downregulation of 39 genes among those who had conceived. Most prominently upregulated genes was for the endometrial bladder transmembrane 1 protein (UPIb), which was found to be localized in the secretory vesicles of the glandular epithelial cells. Among the other genes, upregulated were genes for MUC1, crystallin alpha B, APOD, and PLA2. The authors hypothesized that the endometrial injury increases the expression of genes necessary for endometrial preparation for implantation.

Spandorfer et al., 2005 [19]; studied the effect of autologous endometrial co-culture (AECC) and endometrial injury on pregnancy rate. Their results indicate that although AECC is a useful adjunct in multiple failed IVF attempts, there is no increase in the outcome when the biopsy is performed in the month prior to IVF cycle. This differed from our study as it was a case-control study and the biopsy was performed once, in the luteal phase.

\subsection{Interpretation}

Before applying the results to other populations and settings, several factors have to be considered, the larger number is needed for more evaluation, if genetic and metabolic embryo analysis available preferred to be done, one application done no need for more endometrial trauma, hysteroscopy should be done in any case before IVF-ET, by using the system of endometrial scratch-suction described in same cycle of IVF-ET at day 5 - 7 some polyps and hazardous chemical (suspected to be present), and in addition to remodeling of immunological system that help embryo implantation.

A single, site-specific hysteroscopic biopsy-induced injury was generated on the posterior endometrium at midline 10 - $15 \mathrm{~mm}$ from the fundus during the D4 - D7 period of the ongoing controlled ovarian hyperstimulation cycle. A site-specific hysteroscopic endometrium injury performed during the ongoing in vitro fertilization (IVF) cycle, instead of injuries received during prior cycles, significantly improves clinical outcomes in patients with repeated implantation failure [20]. In this study used of endomtriach scratch suction in same cycle.

The injured endometrium by simple curettage or copper wire could be fully repaired. While the endometrial 
regeneration was severely impaired by double-injury, both areas of endometrium and uterine cavity decreased $(P$ $<0.05)$; both PIs of glandular epithelial and stromal cells increased and reached maximum at 4 weeks $(P<0.05)$, but returned by 8 weeks. A rabbit model of endometrial injury could be effectively established through a doubleinjury procedure of curettage and copper wire with comparable clinical index [21]. In this study not done in animals.

The evidence is strongly in favour of inducing local endometrial injury in the preceding cycle of ovarian stimulation to improve pregnancy outcomes in women with unexplained RIF. However, large randomized studies are required before iatrogenic induction of local endometrial injury can be warranted in routine clinical practice. Some women undergoing IVF treatment fail to conceive despite several attempts with good-quality embryos and no identifiable reason. We call this "recurrent implantation failure" (RIF) where the embryo fails to embed or implant within the lining of the womb. Studies have shown that inducing injury to the lining of the womb in the cycle before starting ovarian stimulation for IVF can help improve the chances of achieving pregnancy. Injury can be induced by either scratching the lining of the womb using a biopsy tube or by telescopic investigation of the womb using a camera. Inducing injury is $70 \%$ more likely to result in a clinical pregnancy as opposed to no treatment. Furthermore, scratching of the lining was 2-times more likely to result in a clinical pregnancy compared with telescopic evaluation of the lining of the womb. In women with RIF, inducing local injury to the womb lining in the cycle prior to starting ovarian stimulation for IVF can improve pregnancy outcomes [22].

So, endometrial scratch in a systematic manner by non traumatizing instrument is associated with higher implantation potential.

By using suction we remove any small polyps in addition to removal of any materials that may be hazardous.

The shortcoming of this trial is the small sample size. A larger study needs to be done to verify the findings and improve the statistical power.

\section{Conclusion}

Here we have demonstrated through an observational prospective cohort experimental study that clinical pregnancy and implantation rates significantly increase after endometrial scratch-suction in same cycle in patients with good-quality embryos. This phenomenon could be due to the injury-induced endometrial decidualization secondary to upregulation of genes encoding for locally acting mediators. Scratch by using insertion tube of intrauterine contraceptive device (IUCD) with suction using infant feeding tube $8 \mathrm{~F}$ after cutting its upper end is an easy and safe outpatient procedure. This certainly needs further investigation.

\section{Acknowledgements}

The author would like to thank all patients included in the study. The author acknowledges the support of the all staff members of Obstetrics and Gynaecology Department Faculty of Medicine Zagazig University for their support.

\section{Contribution to Authorship}

W. S. contributed to the protocol, co-ordinated the study, interviewed the parents, analysed the data and drafted the article. W. S. contributed also to the revision and final approval of the article.

\section{Details of Ethics Approval}

The study received approval from the National Research Ethics Committee Zagazig University, Faculty of medicine. Reference: 29/5/0947. Date of approval: 5 April 2011.

\section{Funding}

All patient included in study at fund of Zagazig University Hospitals which under the funding of ministry of higher education in Egypt.

\section{References}

[1] Dey, S.K., Lim, H., Das, S.K., Reese, J., Paria, B.C., Daikoku, T., et al. (2004) Molecular Cues to Implantation. Endo- 
crine Reviews, 25, 341-373. http://dx.doi.org/10.1210/er.2003-0020

[2] Minas, V., Loutradis, D. and Makrigiannakis, A. (2005) Factors Controlling Blastocyst Implantation. Reproductive BioMedicine Online, 10, 205-216. http://dx.doi.org/10.1016/S1472-6483(10)60942-X

[3] Baum, M., Yerushalmi, G.M., Maman, E., Kedem, A., Machtinger, R., Hourvitz, A., et al. (2012) Does Local Injury to the Endometrium before IVF Cycle Really Affect Treatment Outcome? Results of a Randomized Placebo Controlled trial. Gynecological Endocrinology, 28, 933-936. http://dx.doi.org/10.3109/09513590.2011.650750

[4] Barash, A., Dekel, N., Fieldust, S., Segal, I., Schechtman, E. and Granot, I. (2003) Local Injury to the Endometrium Doubles the Incidence of Successful Pregnancies in Patients Undergoing in Vitro Fertilization. Fertility and Sterility, 79, 1317-1322. http://dx.doi.org/10.1016/S0015-0282(03)00345-5

[5] Granot, I., Gnainsky, Y. and Dekel, N. (2012) Endometrial Inflammation and Effect on Implantation Improvement and Pregnancy Outcome. Reproduction, 144, 661-668. http://dx.doi.org/10.1530/REP-12-0217

[6] Narvekar, S.A., Gupta, N., Shetty, N., Kottur, A., Srinivas, M. and Rao, K.A. (2010) Does Local Endometrial Injury in the Nontransfer Cycle Improve the IVF-ET Outcome in the Subsequent Cycle in Patients with Previous Unsuccessful IVF? A Randomized Controlled Pilot Study. Journal of Human Reproductive Sciences, 3, 15-19. http://dx.doi.org/10.4103/0974-1208.63116

[7] Veeck, L. (1990) The Morphological Assessment of Human Oocytes and Early Conception. In: Keel, B.A. and Webster, B.W., Eds., Handbook of the Laboratory Diagnosis and Treatment of Infertility, CRC Press, Boca Raton, 353369.

[8] Loeb, L. (1907) The Experimental Proof Changes in the Uterine Decidua of Guinea Pig after Mating. Zentralblatt für Allgemeine Pathologie, 18, 563-565.

[9] Humphrey, K.W. (1969) Interaction between Estrogen-17 $\beta$ and Progesterone on the Induction of Deciduomata in Ovariectomized Mice. Australian Journal of Biological Sciences, 22, 689-699.

[10] Finn, C.A. and Martin, L. (1972) Endocrine Control of the Timing of Endometrial Sensitivity to a Decidual Stimulus. Biology of Reproduction, 7, 82-86.

[11] Almog, B., Shalom-Paz, E., Dufort, D. and Tulandi, T. (2010) Promoting Implantation by Local Injury to the Endometrium. Fertility and Sterility, 94, 2026-2029. http://dx.doi.org/10.1016/j.fertnstert.2009.12.075

[12] Raziel, A., Schachter, M., Strassburger, D., Berno, O., Ron-El, R. and Friedler, S. (2007) Favorable Influence of Local Injury to the Endometrium in Intracytoplasmic Intracytoplasmicsperm Injection Patients with High-Order Implantation Failure. Fertility and Sterility, 87, 198-201. http://dx.doi.org/10.1016/j.fertnstert.2006.05.062

[13] Karimzadeh, M.A., Ayazi Rozbahani, M. and Tabibnejad, N. (2009) Endometrial Local Injury Improves the Pregnancy Rate among Recurrent Implantation Failure Patients Undergoing in Vitro Fertilisation/Intra Cytoplasmic Sperm Injection: A Randomised Clinical Trial. Australian and New Zealand Journal of Obstetrics and Gynaecology, 49, 677-680. http://dx.doi.org/10.1111/j.1479-828X.2009.01076.X

[14] Dekel, N., Gnainsky, Y., Granot, I. and Mor, G. (2010) Inflammation and Implantation. American Journal of Reproductive Immunology, 63, 17-21. http://dx.doi.org/10.1111/j.1600-0897.2009.00792.x

[15] Zhou, L., Li, R., Wang, R., Huang, H.X. and Zhong, K. (2008) Local Injury to the Endometrium in Controlled Ovarian Hyperstimulation Cycles Improves Implantation Rates. Fertility and Sterility, 89, 1166-1176. http://dx.doi.org/10.1016/j.fertnstert.2007.05.064

[16] Lessey, B.A. (1999) Integrins and Uterine Receptivity. In: Carson, D.D., Ed., Embryo Implantation: Molecular, Cellular and Clinical Aspects, Springer, New York, 210-222. http://dx.doi.org/10.1007/978-1-4612-1548-6_18

[17] Hurskainen, T., Seiki, M., Apte, S.S., Syrjakallio-Ylitalo, M., Sorsa, T., Oikarinen, A., et al. (1998) Production of Membrane-Type Matrix Metalloproteinase-1 in Early Human Placenta: A Possible Role in Placental Implantation? Journal of Histochemistry \& Cytochemistry, 46, 221-229. http://dx.doi.org/10.1177/002215549804600211

[18] Kalma, Y., Granot, I., Gnainsky, Y., Or, Y., Czernobilsky, B., Dekel, N., et al. (2009) Endometrial Biopsy-Induced Gene Modulation: First Evidence for the Expression of Bladder Transmembranal Uroplakin Ib in Human Endometrium. Fertility and Sterility, 91, 1042-1049. http://dx.doi.org/10.1016/j.fertnstert.2008.01.043

[19] Spandorfer, S.D., Delgado, Y., Park, J., Clark, R. and Rosenwaks, Z. (2005) The Success in IVF When Utilizing Autologous Endometrial Coculture (AECC) Is Not Secondary to a Local Endometrial Injury. Fertility and Sterility, 84, S52-S53. http://dx.doi.org/10.1016/j.fertnstert.2005.07.124

[20] Huang, S.Y., Wang, C.J., Soong, Y.K., Wang, H.S., Wang, M.L., Lin, C.Y., et al. (2011) Site-Specific Endometrial Injury Improves Implantation and Pregnancy in Patients with Repeated Implantation Failures. Reproductive Biology and Endocrinology, 9, 140. http://dx.doi.org/10.1186/1477-7827-9-140

[21] Li, L., Shi, J., Zhang, Q.F., Yan, J., Yan, L.Y., Shen, F., et al. (2011) Effect of Curettage and Copper Wire on Rabbit Endometrium: A Novel Rabbit Model of Endometrial Mechanical Injury. Chinese Medical Journal, 124, 1708-1713. 
[22] Potdar, N., Gelbaya, T. and Nardo, L.G. (2012) Endometrial Injury to Overcome Recurrent Embryo Implantation Failure: A Systematic Review and Meta-Analysis. Reproductive BioMedicine Online, 25, 561-571.

http://dx.doi.org/10.1016/j.rbmo.2012.08.005 